\title{
On displacement-based plastic design of parallel chord vierendeel girders
}

\author{
Mark Grigorian
}

Received: 31 March 2014 / Accepted: 18 June 2014/Published online: 19 July 2014

(c) The Author(s) 2014. This article is published with open access at Springerlink.com

\begin{abstract}
The paper introduces the principles of displacement-based plastic design (DBPD) and its applications to the efficient design of parallel chord steel vierendeel girders under normal nodal forces. A simplifying assumption has been made that the mathematical model is composed of imaginary, pin connected modules that fit within the bays of the prototype. The use of this modeling concept in conjunction with the applications of the uniform strength theory leads to the development of an algorithm that is ideally suited for manual, minimum weight design of steel vierendeel girders under any distribution of vertical nodal forces. The resulting solutions are exact and unique and lend themselves well to DBPD and minimum weight treatment. In DBPD which is akin to performance control, member strengths and stiffnesses are assigned rather than tested. Several generic examples have been provided to illustrate the applications of the proposed design procedures. The numerical results of these examples have been verified through long hand and computer methods of analysis. An extensive proof of the proposed method of approach has been provided in the "Appendix".
\end{abstract}

Keywords Vierendeel girders · Plastic design .

Minimum weight . Displacements at incipient collapse

\section{Introduction}

Plastic design is the basis of a number of recently developed design methodologies for moment frames under

M. Grigorian $(\bowtie)$

MGA Structural Engineering Consultants Inc.,

Glendale, CA, USA

e-mail: markarjan@aol.com lateral loading, (Mazzolani and Piluso 1997; Goel et al. 2010; Grigorian and Grigorian 2011). The technical merits and economic benefits of plastic design have been amply documented in the literature as well as such authoritative texts such as Baker et al. (1956), Beedle (1958), Neal (1963), Nethercot (2001). Plastic design is also the cornerstone of the present contribution. The novelty of the present contribution is in that it incorporates the magnitude and location of maximum plastic deformations as part of the member sizing criteria. A prior knowledge of the elastoplastic performance of any structure at distinct stages of loading, such as at first yield and incipient collapse, may help design the system as a structure of uniform strength (US). Structures of US lend themselves well to DBPD, PC and direct material optimization. A large number of regular structural frameworks such as moment frames, (Wong 2009; Grigorian and Grigorian 2012a), trusses, grillages, vierendeel girders (VG) etc., can be designed and/or optimized as structures of US. Since the demand-capacity ratio is constant for the constituent members of structures of US, they tend to fail in a state of over-complete collapse where all plastic hinges form simultaneously. While the state of over-complete collapse is associated with minimum weight design, the corresponding distribution of plastic hinges can be looked upon as an admissible template for imposing any order of propagation of plasticity without affecting the economics and/or the ultimate carrying capacity of the system.

The currently available design and optimization techniques are highly iterative in nature and can best be utilized if the corresponding software is accessible. All such procedures begin with an initial design followed by numerous iterations, until convergence takes place. The problem constraints consist usually of sets of code and purposespecific conditions. Since both the original and the final 
(optimized) designs are addressed through iterative processes, the combined effort may be viewed as prospective in nature and investigative in effort, and as such apply to almost all traditional designs for contemporary structures. In contrast to prospective processes that seek to satisfy sets of prescribed criteria, knowledge-based methodologies are expected to meet certain response objectives. In investigative procedures, theories of structures and material science are followed rather than applied. It might interest the reader to note that in the making of natural structures, such as trees, knowledge is induced rather than tested.

The purpose of this paper is to introduce DBPD as an observation-based approach for the efficient design of certain types of moment frames such as parallel chord VG and similar structures, (Grigorian 2014). Performance-oriented designs are knowledge-based strategies that aim at providing insight into the response of certain types of structures under specific loading conditions. In this context, observation based refers to reliance on theoretical and/or experimental data for realistic design purposes. DBPD is a recent development that allows for the imposition of the required as well as desirable design requirements, as opposed to investigating the same as results of iterative computations. The desirable requirements may include the ability to control the sequences of formations of the plastic hinges, limit the maximum displacements at incipient collapse, optimize the self weight of the structure, prevent premature collapse, etc. DBPD is inherently capable of resulting in minimum weight elastoplastic solutions for ductile moment frames and as such provides an opportunity for the efficient design of steel VG under in-plane loading.

The paper begins by reviewing the fundamental concepts involved in modular analysis of regular moment frames and structure-specific findings that help formulate the proposed solutions. The limit state load carrying characteristics of parallel chord VG of US under arbitrary distribution of normal nodal forces are then presented as a prelude to the determination of the efficient design of $\mathrm{VG}$ through several generic examples. These examples present a number of useful formulae for the preliminary design of VG. All symbols are defined as they first appear in the text. The paper does not address the vibratory behavior of $\mathrm{VG}$ (Grigorian 1970).

\section{The modeling concept}

It has been demonstrated by Grigorian (1993), Grigorian and Grigorian (1988), Taranath (1998) and Bozorgnia and Bertero (2004) that mathematical models of upright, regular rectangular moment frames can be construed as being composed of hypothetical, rigidly jointed, rectangular modules stacked on top of each other and supported on base level boundary modules. A similar approach has been adopted here to study the efficient design of VG as horizontal moment frames, simply supported at both ends and subjected to a monotonically increasing distribution of normal nodal forces applied along the top and/or bottom chords of the girder. The effects of shear, panel zone, axial load and other secondary phenomena on flexural response of the system have been discarded for the sake of simplicity. Strain hardening and plastic hinge offsets have also been ignored in favor of clarity as well as higher load factors against failure.

The pioneering studies pertaining to plastic design of VG are due to Hendry (1955) and Morovich (1958) who introduced the applications of simple plastic theory to the design of mild steel parallel chord VG. The line diagram of a generalized parallel chord $\mathrm{VG}$ under an arbitrary distribution of normal nodal forces is presented in Fig. 1a. The corresponding mathematical model composed of imaginary rectangular modules connected to each other in series by means of hypothetical rigid links is shown in Fig. 1b. Coordinates $i=0,1,2 \ldots n$ refer to the locations of the joints of the girder along its horizontal axis. The entire structure is composed of $n+1$ vertical, and $2 n$ horizontal members. The location of each module is identified by the coordinate of its right hand joint. Here, $J$ and $I$, and $N$ and $M$ stand for section moduli and bending moments, respectively, of the beams and posts of the original structure. Similarly, $\bar{J}$ and $\bar{I}$, and $\bar{N}$ and $\bar{M}$ represent the section moduli and bending moments, respectively, of the beams and posts of imaginary modules of Fig. 1b. These modules are forced to deform compatibly and to remain in equilibrium throughout the loading history of the structure. Obviously, when the imaginary modules are merged to reconstruct the prototype, the hypothetical links disappear and the moments of inertia and bending moments of the beams and columns of the reassembled structure become:

$M_{i}=\bar{M}_{i}+\bar{M}_{i+1}, N_{i}=\bar{N}_{i}=\bar{M}_{i}$ and $I_{i}=\bar{I}_{i}+\bar{I}_{i+1}, J_{i}=\bar{J}_{i}$

By the same token, the plastic moments of resistance of the merging modules or the reassembled structure may be expressed as:

$M_{i}^{P}=\bar{M}_{i}^{P}+\bar{M}_{i+1}^{P}$ and $N_{i}^{P}=\bar{N}_{i}^{P}=\bar{M}_{i}^{P}$

It follows therefore that $M_{0}^{P}=N_{1}^{P}=\bar{M}_{1}^{P}, M_{n}^{P}=N_{n}^{P}=$ $\bar{M}_{n}^{P}, J_{1}=\bar{J}_{1}, I_{0}=\bar{I}_{1}, J_{n}=\bar{J}_{n}$ and $I_{n}=\bar{I}_{n}$.

The basic module

The premise of the concept is that if the individual modules can be selected to satisfy the prescribed performance criteria, then their assemblage should also be in conformance 

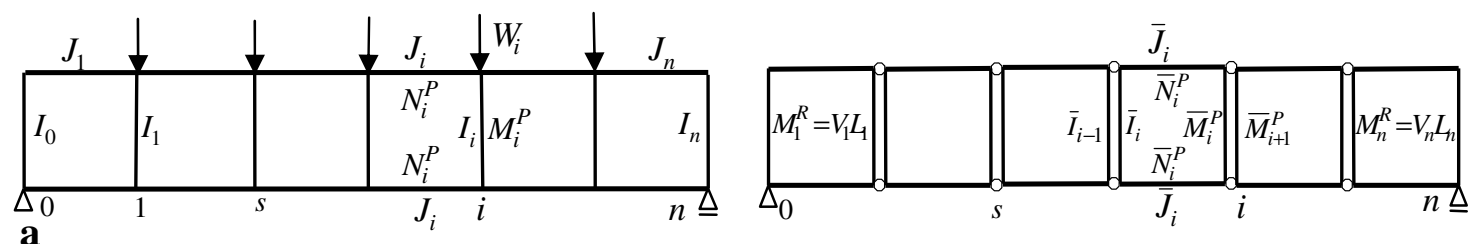

b

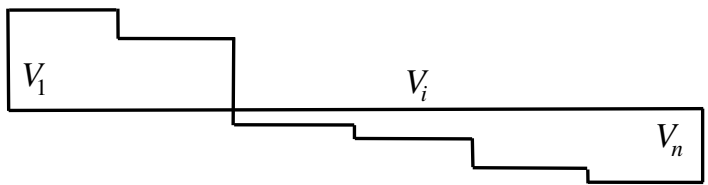

c

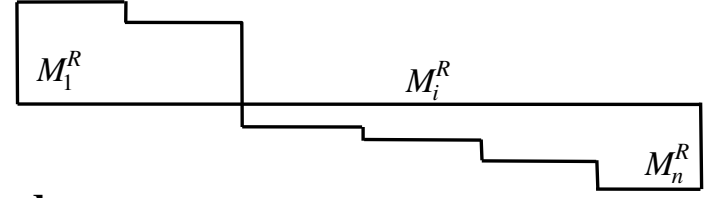

d

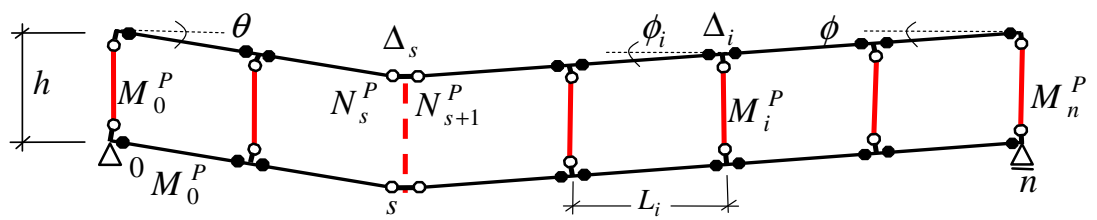

e

Fig. 1 a Parallel chord vierendeel girder. b Imaginary pin connected model. c Shear force diagram. d Racking moment diagram. e Failure mechanism; vierendeel girder of uniform strength including active open circle and filled circle inactive hinges

with the stipulated design conditions. In other words, if each module is designed to be of minimum weight with respect to the expected mode of response, then their assembly would also be of minimum weight with respect to the same performance requirements. Some of the fundamental observations, leading to the development of the proposed algorithm have been summarized as follows, that;

- "In cases where all design variables can be calculated from the condition of US, the US design and minimum weight/volume design coincide" (Brandt 1984).

- "Modular modeling can reduce the task of otherwise complex structural analysis to simple manual computations" (Bozorgnia and Bertero 2004).

- Doubly symmetric rectangular frame modules, subjected to uniform nodal shears can be designed as minimum weight, US systems (Grigorian 1993).

- Rectangular modules of US may be combined horizontally and/or vertically to form new load bearing assemblies of US, (Grigorian and Grigorian 2012b, c).

- Individual modules are quasi-determinate sub-assemblies and provide great insight into the performance of the structure during the design process.

- The end sections of the members of the module can be treated in such a way as to prevent or force the formation of plastic hinges at those locations, e.g., using reduced beam sections, added flange plates, haunches, etc. This implies that the locations and sequences of formations of the plastic hinges can be controlled as intended.
- The moments and deformations of the elements of the VG of US at any ordinate $i$ may be related to the local racking moment $M_{i}^{R}=V_{i} L_{i}$, Fig. 1d, of the corresponding module, e.g., $\bar{M}_{i}=M_{i}^{R} / 4$, where $V_{i}$ is the total external shear acting on joint $i$, Fig. $1 \mathrm{c}$.

- The stiffnesses of the individual modules can be selected in such a way as to control the maximum displacements of the frame throughout the elastoplastic loading history of the frame.

- Due to double symmetry, the points of inflections of the vertical elements of parallel chord girders occur at their mid heights.

- In structures of US, the redistribution of moments through formation of plastic hinges forces the corresponding points of inflection to move towards the mid spans of the beams.

- VG of US may fail through a state of over collapse, where all constituent modules fail together.

- While steel moment frames under lateral loading are most suitable for plastic design, they are also susceptible to unfavorable effects of drift shifting, especially at incipient collapse. Hamburger et al. (2009) and Grigorian (2013b) have reported that a reduction in differential drift can help improve racking stability in all categories of moment frames.

Hence, an observed assertion was made that points of inflections of all members of VG of US occur at their mid spans during the elastic as well as plastic stages of loading. 
This is an acceptable presumption with inconsequential margins of error since the solution becomes more accurate and eventually exact as plasticity spreads over all members of the structure. These observations form the essence of the optimization philosophies that lead to the minimum weight design of regular moment frames including parallel chord VG. Likewise, the indispensible association of the mid-span points of inflection with the exact minimum weight design conditions immensely facilitates the comprehension and mathematical formulation of the problem. In addition, an observation has been made that in almost all types of traditionally designed frameworks, the drift angle due to racking effects is generally a maximum near the supports and a minimum near the location of maximum displacement (Taranath 1998). This suggests that it would be prudent to first alleviate the possibility of premature formation of plastic hinges within the first and last bays of the frame, minimize large drift angles near the supports and then work sideways to control the displacements of the remaining bays or modules. The anticipated single collapse mechanisms of structures of US have more than one degree of kinematic freedom and can be construed as being composed of several merging failure patterns. Structures of US almost invariably fail through a state of over-complete collapse. The likelihood of over-complete collapse, suggests that the sequences of formations of the plastic hinges may be controlled in such a way as to prevent the formation of plastic hinges in the vertical members until the first sets of hinges form in the desired locations of the horizontal elements. Similarly it would be possible to delay the formation of plastic hinges at the critical ends of the horizontal members until the complete failure of all vertical members. Moment control technologies, such as added flange plates and/or reduced beam sections can be employed to induce any mode of collapse and sequence of formation of plastic hinges, without noticeably affecting the ultimate load carrying and/ or displacement development capacity of the structure.

\section{Theoretical development}

The purpose of this section is to utilize the information presented in the preceding sections to design a generalized parallel chord VG of US under an arbitrary distribution of normal nodal forces. This is achieved by assigning as much strength and stiffness as demand imposed on each module of the structure. The strength $N_{i}^{P}$ of a module is defined as its maximum carrying capacity at failure. The minimum stiffness of the module is directly related to its target drift ratio $\theta$ at incipient collapse. $\theta$, and its compatible counterpart $\phi$, (Fig. 1e) are the two drift angles that uniquely define the location $s$ of the maximum nodal displacement $\Delta_{s}$ at collapse as well as the correct collapse mode of the framework. Since $\theta_{i}=\theta$ for $0 \leq i \leq s$ and $\theta_{i}=\phi_{i}=\phi$ for $s \leq i \leq n$ then $\theta$ and $\phi$ may be related to each other through $\Delta_{s}=\theta \sum_{i=1}^{s} L_{i}=\phi \sum_{i=s+1}^{n} L_{i}$.

Capacity assignment, strength design

Perhaps the most interesting facet of modular structures of US is that the ultimate carrying capacities of their members can be estimated almost by inspection (Grigorian and Grigorian 2012a, d, e). This leads to the statement that: the maximum carrying capacity of any module $i$ of a vierendeel girder of US can be related directly to the ultimate racking moment acting on that module. In other words, once the external shear and racking moment diagrams of a generalized, parallel chord vierendeel girder such as those depicted in Fig. 1c, d are known, the corresponding exact capacities may be computed as:

$N_{i}^{P}=\frac{M_{i}^{R}}{4}=\frac{V_{i} L_{i}}{4}$.

The validity of the distribution of $N_{i}^{P}$ may be verified by the sum $\sum_{i=1}^{n} V_{i}=4 \sum_{i}^{n}\left(N_{i}^{R} / L_{i}\right)=0$. While there is no need to substantiate the soundness of Eq. (3), it is instructive to ascertain the plausibility of the postulated collapse mechanism. The failure mode of Fig. 1e indicates that due to the development of $4(n-1)$ inactive plastic hinges along the chords of the structure, the girder may tend to fail through a state of over-complete collapse with only two sets of active plastic hinges along the chords of the structure. The inactive hinges are shown as black solid circles. This allows the virtual work equation of the selected failure scenario to be expressed as:

$\sum_{i=0}^{n} W_{i} \Delta_{i}=2 \theta N_{s}^{P}+2 \phi N_{s+1}^{P}+2 \theta \sum_{i=0}^{s-1} M_{i}^{P}+2 \phi \sum_{i=s+1}^{n} M_{i}^{P}$

or, as:

$$
\begin{aligned}
\sum_{i=0}^{n} W_{i} \Delta_{i}= & 2 \sum_{i=0}^{s}\left[\left(1-\delta_{i}^{s}\right) M_{i}^{P}+\delta_{i}^{s} N_{i}^{P}\right] \theta \\
& +2 \sum_{i=s+1}^{n}\left[M_{i}^{P}+\delta_{i}^{s+1} N_{i}^{P}\right] \phi=2 \sum_{i=1}^{n} M_{i}^{P} \theta_{i}
\end{aligned}
$$

where the Kroneckar's delta $\delta_{i}^{s}$ has been introduced to override the mathematical complexities arising from abrupt changes in plastic rotations $\theta_{i}$ and $\phi_{i}$. Here, $\delta_{i}^{s}=1$ for $i=s, \quad \delta_{i}^{s}=0$ for $i \neq s, \quad\left(1-\delta_{i}^{s}\right)=0$ for $i=s$ and $\left(1-\delta_{i}^{s}\right)=1$ for $i \neq s$. The virtual work equation corresponding to the same failure mechanism but in terms of the single variables $\bar{M}_{i}^{P}$ of the collapsing modules may be expressed as: 
$\sum_{i=1}^{n} W_{i} \Delta_{i}=4 \sum_{i=1}^{n} \bar{M}_{i}^{P} \theta_{i}=2 \sum_{i=0}^{n}\left(\bar{M}_{i}^{P}+\bar{M}_{i+1}^{P}\right) \theta_{i}=2 \sum_{i=1}^{n} M_{i}^{P} \theta_{i}$

Since, Eqs. (5) and (6) are identical then the modular concept can be expected to yield the same exact results as the traditional methods of approach.

\section{Demonstrative example 1}

Design a generalized vierendeel girder of US with $n$ identical bays of height $h$ and length $L$ under a uniform distribution of normal nodal forces $W$.

Solution while there is no need to conduct a virtual work analysis to determine the plastic moments of resistance of each module, an understanding of the deformed shape of the structure is essential to developing insight into the performance of the structure and estimating the corresponding displacements at incipient collapse. The admissible collapse mechanisms of the subject VG for even and odd numbers of bays are depicted in Fig. 2a, b, respectively. Because of double symmetry only the solution of the left half of the girder is addressed in the present section.

The exact minimum weight solutions for both the even and odd number of bays can be written down, either by inspection or following the guidelines of the preceding section. The vertical shear $V_{i}$ sustained by each module may be expressed as a continuous function of the variable $i$ as:

$V_{i}=(n+1-2 i) \frac{W}{2}$ for $i=1,2,3 \ldots n$.

this gives:

$$
\begin{aligned}
& M_{0}^{P}=\bar{M}_{0}^{P}=N_{1}^{P}=\bar{M}_{n}^{P}=M_{n}^{P}=N_{n}^{P}=(n-1) \frac{\mathrm{WL}}{8} \\
& \bar{M}_{i}^{P}=N_{i}^{P}=\frac{M_{i}^{R}}{4}=\frac{V_{i} L_{i}}{4}=(n+1-2 i) \frac{\mathrm{WL}}{8} \\
& \text { for } i=1,2,3 \ldots . .
\end{aligned}
$$

Fig. 2 Regular parallel chord vierendeel girder under uniform loading. a Admissible failure mechanism, $n$ even. b Admissible failure mechanism, $n$ odd

Fig. 3 Regular parallel chord vierendeel girder under arbitrarily placed point loading. a Geometry and failure mechanism. b Racking moment diagram
$M_{i}^{p}=\bar{M}_{i}^{P}+\bar{M}_{i+1}^{P}=(n-2 i) \frac{\mathrm{WL}}{4}$ for $i=1,2,3 \ldots n-1$

The validity of solutions (8), (9) and (10) may be verified through the corresponding work equation; $W_{\text {ext. }}=$ $W_{\text {int. }}$. Assuming $\Delta_{i}=i \times \Delta$ and, $\Delta=L \times \theta$, it gives:

$W_{\text {ext. }}=2 \sum_{i=1}^{n-1 / 2} W \Delta_{i}=\left[2 n^{2}+(-1)^{n}-1\right] \frac{\mathrm{WL} \theta}{8}$,
$W_{\text {int. }}=2 \sum_{i=1}^{n-1 / 2} 4 M_{i}^{P} \theta=4 \theta\left[2 n^{2}+(-1)^{n}-1\right] \frac{\mathrm{WL}}{32}$,

\section{Demonstrative example 2}

Design a generalized VG of US with $n$ identical bays of height $h$ and length $L$ under a concentrated normal nodal force $P$ at an arbitrary joint $j$.

Solution the postulated collapse mode of the girder under consideration is presented in Fig. 3. The problem is essentially that of solving a structurally determinate system. However, assuming that the postulated failure mechanism of Fig. 3a is correct then the corresponding racking moment diagram may be presented as Fig. 3b, whence;

$\bar{M}_{i}^{P}=N_{i}^{P}=\frac{M_{i}^{R}}{4}=\frac{V_{i} L}{4}=\left[\frac{(n-s)}{n}-\langle i-s\rangle^{0}\right] \frac{\mathrm{PL}}{4}$

In other words $\bar{M}_{i}^{P}=N_{i}^{P}=M_{i}^{R} / 4=(n-s) \mathrm{PL} / 4 n$ for $1 \geq i \geq s$ and $\bar{M}_{i}^{P}=N_{i}^{P}=M_{n}^{R} / 4=s \mathrm{PL} / 4 n$ for $(s+1) \geq$ $i \geq n$. The complete solution for the vertical members may now be summarized as:

$$
\begin{aligned}
M_{i}^{p}= & \bar{M}_{i}^{P}+\bar{M}_{i+1}^{P}=\left[\frac{2(n-s)}{n}-\langle i-s\rangle^{0}-\langle i+1-s\rangle^{0}\right] \\
& \times \frac{\mathrm{PL}}{4} \text { for } i=1,2,3, \ldots n-1
\end{aligned}
$$

Here, the step function $\langle i-s\rangle^{0}=0$ for $i<s$ and $\langle i-s\rangle^{0}=1$ for $i>s$. For the particular case $n=2 s$, Eq. (12) reduces to $\bar{M}_{i}^{P}=N_{i}^{P}=\mathrm{PL} / 8$.

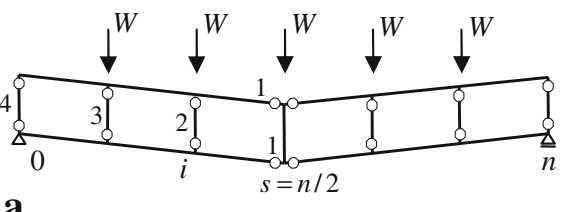

$\mathbf{a}$

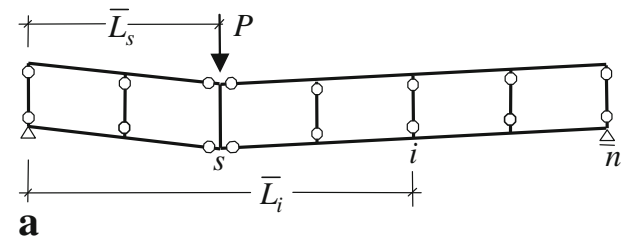

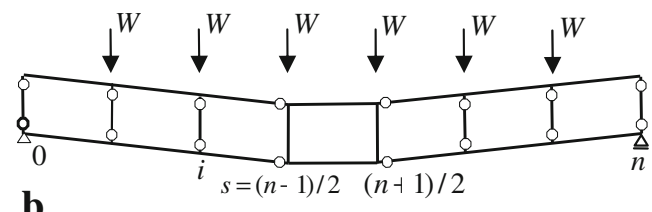

b 


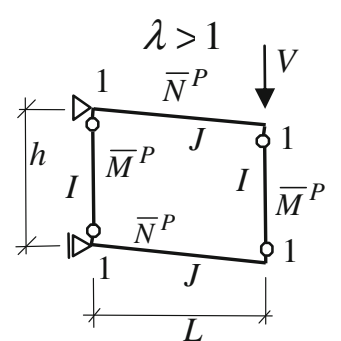

a

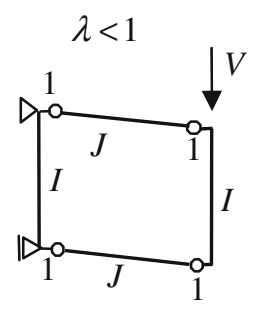

b

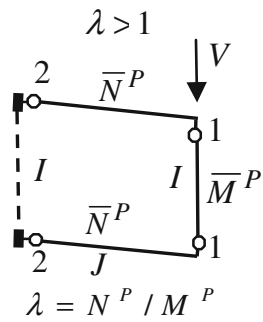

c

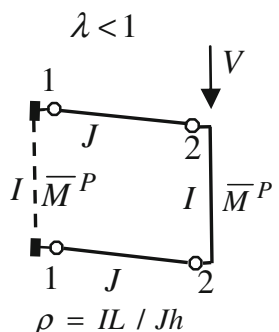

d

Fig. 4 a, b Rectangular closed loop modules at collapse, c, d Portal type modules at collapse

\section{Displacements at incipient collapse}

The inclusion of maximum displacements at incipient collapse helps develop insight into the response of the structure at incipient collapse. The deformed shape of the collapsing VG of Fig. 1e may be construed as being composed of a number of compatible deformed modules that fit into the bays of the structure. Comparing the geometric dispositions of the modules of Fig. 1e with the deformed shapes of Fig. 4, it may be seen that the two adjoining modules at joint $s$ tend to change shape as end restrained modules of Fig. 4c, d, whereas all other modules tend to deform as unrestrained rectangular frames such as those depicted in Fig. 4a, b. It has been shown, through the use of Eqs. (1-13), that the computational model of Fig. 1b can be utilized to accurately estimate the ultimate load carrying capacities of parallel chord, VG of US, without resorting to complicated structural analysis. An attempt has been made in the present section to show that a similar approach may be adopted to study the corresponding maximum normal nodal displacements at incipient collapse.

Girder displacements at incipient collapse

The generalized force deformation relationship of typical VG, such as that shown in Fig. 1a or e, in terms of module stiffness ratios $\rho_{i}=I_{i} L_{i} / h J_{i}$, and corner moments $M_{i}$ or $\bar{M}_{i}^{P}$ may be expressed as:

$$
\begin{aligned}
\Delta= & \sum_{i=1}^{s-1} \frac{M_{i} L_{i}^{2}}{6 E J_{i}}\left(1+\frac{1}{\rho_{i}}\right)+\frac{M_{s} L_{s}^{2}}{12 E J_{s}}\left(2+\frac{1}{\rho_{s}}\right) \\
& +\frac{\left(M_{s}-M_{s+1}\right) h L_{s}}{12 E\left(I_{s}+I_{s+1}\right)}
\end{aligned}
$$

or,

$$
\begin{aligned}
\Delta= & \frac{\left(M_{s}-M_{s+1}\right) h L_{s+1}}{12 E\left(I_{s}+I_{s+1}\right)}+\frac{M_{s+1} L_{s+1}^{2}}{12 E J_{s+1}}\left(2+\frac{1}{\rho_{s+1}}\right) \\
& +\sum_{i=s+2}^{n} \frac{M_{i} L^{2}}{6 E J_{i}}\left(1+\frac{1}{\rho_{i}}\right)
\end{aligned}
$$

In other words, if it can be shown that each term of Eqs. $(14,15)$ defines the lateral displacement of an independent module, then it may be suggested that; the maximum normal nodal displacements of VG of US at incipient collapse may be computed as the sum of the corresponding displacements of the individual modules either to the left or right of the joints of maximum plastic rotation. This implies that both the left and right hand segments of the collapsing girder may be used as suitable primary structures for displacement analysis (Hayman 1961, 1971; Horne 1979). A formal proof of Eqs. $(14,15)$, together with an example utilizing both segments of the girder for displacement analysis is also presented in the "Appendix". Computations based on Eqs. $(14,15)$ satisfy the prescribed yield criteria, static equilibrium, boundary support conditions as well as the kinematics of the postulated failure mechanism, and as such satisfy the requirements of the uniqueness theorem and minimum weight design due to Foulkes $(1953,1954)$.

Module displacements at incipient collapse

The study of the maximum displacements of basic modules as constituent elements of VG at incipient collapse may help appreciate the essence of the proposed procedures. Two local deformability conditions affect the global displacements of the vierendeel frame. Depending upon their location, neighboring modules can deform and rotate either in the same or opposite directions. Module rotations are caused due to changes in plastic curvatures or as dictated by the boundary support conditions. The numerals 1 and 2 in the purely sway collapse mechanisms of Fig. 4 refer to the sequences of formation of the plastic hinges. The dashed lines of Figs. 1e, $4 \mathrm{c}, \mathrm{d}$ represent specific locations where the curvature tends to reverse direction. The dashed line elements tend to remain elastic and render the modules effectively fix ended. For fixed end modules, the sequence of formation of the hinges depends on the over-strength factor $\lambda$ as well as the relative stiffness $\rho$ of the module. In case of modules of US, 
i.e., $\lambda=1$, plastic hinges can form at the four corners simultaneously.

Unrestrained rectangular modules

Unrestrained modules can deform freely in accordance with failure modes (a) and (b) of Fig. 4. For modules rotating in the same direction, failure may occur through the simultaneous formation of plastic hinges in either the vertical or horizontal members of the module. An inspection of the first and last terms of Eqs. $(14,15)$ indicates that the displacements of doubly symmetric rotationally unrestrained modules at incipient collapse may be expressed as:

$\delta=\left[1+\frac{1}{\rho}\right] \frac{M L^{2}}{6 E J}, M=M^{P}=\frac{\mathrm{VL}}{4}$ for $\lambda \geq 1$ and

$M=N^{P}=\frac{\mathrm{VL}}{4}$ for $\lambda<1$

End restrained rectangular modules

Partially end restrained and fixed base portal modules may be used advantageously to address the plastic compatibility conditions where curvatures change sign. The sway mechanisms of Fig. 4c, d depict two distinct possibilities depending upon the relative values of $\rho$ and $\lambda$. The dashed line in Fig. 2e represents the location of two back to back, imaginary fixed base portal frames on their sides. However, since plastic rotations change sign at the sides of the joints that contain the maximum plastic displacement, the common vertical post at $s$ remains elastic and causes the adjoining modules to act as rotationally restrained horizontal portal frames. However, for restrained end modules of US, with $\lambda=1$ the maximum lateral displacement at collapse may be computed using the last two terms of Eq. (14), i.e.,

$\delta=\frac{M_{s} L_{s}^{2}}{12 E J_{s}}\left(2+\frac{1}{\rho_{s}}\right)+\frac{\left(M_{s}-M_{s+1}\right) h L_{s}}{12 E\left(I_{s}+I_{s+1}\right)}$

The second term of Eq. (17) contains the form and magnitude of the curvature of the common vertical member needed to maintain deformation compatibility between the two adjoining modules. Two particular cases are frequently encountered. First, $M_{s}=M_{s+1}=M^{P}$, or $I_{s+1}=\infty$, i.e., cases that describe the displacements of fully fixed portal frames at incipient collapse, whence:

$\delta=\left[2+\frac{1}{\rho}\right] \frac{M L^{2}}{12 E J}, M=M^{P}=\frac{\mathrm{VL}}{2(1+\lambda)}$

Second, $M_{s+1}=I_{s+1}=0$, reduces Eqs. (17) to (16). A detailed account of the plastic displacement analysis of portal frames under combined gravity and lateral forces, including the $p$ delta effects, with different boundary support conditions may be found in Grigorian and Grigorian (2012b, e).

\section{Demonstrative example 3}

Estimate the maximum normal nodal displacement of the vierendeel girder of example 1 at incipient collapse. Assume the chords and the verticals of the girder are constructed out of sections of inform moments of inertia $J$ and $I_{1}=I_{n}$ and $I_{i}=2 I$ for all other $i$, respectively, and that US is achieved by appropriately detailed reduced beam section segments.

Solution it is convenient to solve for $n$ even and odd cases separately. Since for $n=$ even, $M_{s}=M_{s+1}$ and $M_{i}=(n+1-2 i) \mathrm{WL} / 8$, Eq. (14) gives:

$$
\begin{aligned}
\Delta & =\sum_{i=1}^{\frac{n}{2}-1} \frac{M_{i} L^{2}}{6 E J}\left(1+\frac{1}{\rho}\right)+\frac{M_{n / 2} L^{2}}{12 E J}\left(2+\frac{1}{\rho}\right) \\
& =\frac{W L^{3}}{96 E J}\left[\frac{n^{2}}{2}\left(1+\frac{1}{\rho}\right)-\frac{1}{\rho}\right]
\end{aligned}
$$

By the same token, since for $n=$ odd $M_{s+1}=$ $I_{s+1}=0$, Eq. (14) yields

$$
\Delta=\sum_{i=1}^{\frac{n-1}{2}} \frac{M_{i} L^{2}}{6 E J}\left(1+\frac{1}{\rho}\right)=\frac{W L^{3}}{192 E J}\left[\left(n^{2}-1\right)\left(1+\frac{1}{\rho}\right)\right]
$$

\section{Demonstrative example 4}

Estimate the maximum normal nodal displacement of the VG of example 2 at incipient collapse.

Member properties are the same as for example 3 above.

Solution since $M_{i}=M_{s}=\mathrm{PL}(n-s) / 4 n$ and, Eq. (14) gives, upon substitution:

$\Delta=\frac{P L^{3}}{96 n E J}\left\{(n-s)\left[4 s\left(1+\frac{1}{\rho}\right)-\frac{1}{\rho}\right]-\frac{s}{\rho}\right\}$

\section{Displacement-based plastic design}

An important aspect of DBPD is its ability to control and relate the performance of the structure to its maximum displacements at incipient collapse. While it is possible to control the displacements of VG through the use of Eqs. (14) and (15), practical or other considerations may stipulate the need for constant sections or uniform inter-panel drift ratios in addition to other limitations. A method of controlling the drift ratios is presented as follows.

Stiffness assignment, displacement control

Assuming that the chords of the girder rotate through constant drift angles $\theta_{i}=\theta$ and $\phi_{i}=\phi$, at collapse, then the maximum nodal displacement at $s$ may be computed as: $\Delta_{s}=\theta \sum_{i=1}^{s} L_{i}=\phi \sum_{i=s+1}^{n} L_{i}, \quad$ or $\quad \Delta_{s}=\sum_{i=1}^{s} \delta_{i}=$ $\sum_{i=s+1}^{n} \delta_{i}$. And, if $\Delta_{s}$ is assigned a target value $\Delta_{s}=\Delta$, 
then $\theta_{i}=\theta=\Delta / \sum_{i=1}^{s} L_{i} \quad$ and $\quad \phi_{i}=\phi=\Delta / \sum_{i=s+1}^{n} L_{i}$. Consequently, to minimize the inter-panel drift, the individual moments of inertias of the individual module may be selected as;

$$
\begin{aligned}
J_{i}= & {\left[1+\frac{1}{\rho_{i}}\right] \frac{M_{i}^{P} L_{i}}{6 E \theta} \text { and } J_{s}=\frac{M_{s} L_{s}^{2}}{12 E \theta} } \\
& \times\left[\left(2+\frac{1}{\rho_{s}}\right)+\frac{\left(M_{s}-M_{s+1}\right)}{\left(1+\eta_{s}\right) \rho_{s}}\right] \text { for } 1 \leq i \leq s \\
J_{i}= & {\left[1+\frac{1}{\rho_{i}}\right] \frac{M_{i}^{P} L_{i}}{6 E \phi} \text { and } J_{s+1}=\frac{M_{s+1} L_{s+1}^{2}}{12 E \phi} } \\
& \times\left[\left(2+\frac{1}{\rho_{s+1}}\right)-\frac{\left(M_{s}-M_{s+1}\right)}{\left(1+\eta_{s+1}\right) \rho_{s+1}}\right] \\
& \quad \text { for } n \leq i \leq s+1
\end{aligned}
$$

where, $\eta_{s}=I_{s+1} / I_{s}$ and $\eta_{s+1}=I_{s} / I_{s+1}$.

\section{Demonstrative example 5}

Select the properties of a three bay regular VG of US subjected to a single concentrated normal nodal force $P$ in such a way that maximum drift does not exceed the prescribed value $\gamma$.

Solution for $n=3$ and $s=1$, Eq. (12) gives; $M_{1}^{P}=2 \mathrm{PL} /$ 12 and $M_{2}^{P}=M_{3}^{P}=\mathrm{PL} / 12$. A detailed analysis of the moments $M_{i}^{P}$ of the current example is provided as part of example 7 of the "Appendix". For $\rho_{1}=1$, Eqs. (17) or (21) give, $\delta_{1}=\Delta_{\text {left }}=13 P L^{3} / 288 E I_{1}$. Consequently, $I_{1}=I=$ $13 P L^{3} / 288 \gamma E$. Similarly, from Eq. (17), $\delta_{2}=5 P L^{3} / 288 E I_{2}$, therefore $I_{2}=5 P L^{2} / 288 \gamma E=5 I / 13$. Next, Eq. (18) gives $\delta_{3}=8 P L^{3} / 288 E I_{3}$, which in turn yields $I_{3}=8 P L^{2}$ / $288 \gamma E=8 I / 13$. The validity of the proposed solution may be verified by observing that $\Delta_{\text {right }}=\delta_{2}+\delta_{3}=P L^{2}$ / $288 \gamma E$. The VG of example may be categorized as a structure of uniform response where uniform strength is also associated with uniform drift (Grigorian 2013a, b).

\section{Control of sequences of formation of plastic hinges}

As the state of over-complete collapse is associated with the simultaneous formation of all plastic hinges, it can be used as a roadmap to induce a controlled, sequential formation of plastic hinges without compromising the limit carrying capacity or the minimum weight status of the framework. In joint loaded $\mathrm{VG}$, the drift angle due to racking effects is generally a maximum near the supports and a minimum within the span. This implies that it would be prudent to prevent the possibility of premature formation of plastic hinges near the supports. The present section proposes a simple method for delaying the formation of plastic hinges near the supports until the rest of the structure tends to become a mechanism. This is achieved by accelerating the sequences of formations of plastic hinges by the implementation of reduced beam sections in a calculated manner and then compensating for the loss of the global carrying capacity of the structure. For instance, if it is desired to accelerate the formation of the plastic hinges of a rotationally active member, such as the horizontal elements to the left of joint $s$ or the vertical element at joint $s-1$ of Fig. 1e, then it would be sufficient to reduce the plastic moment of resistance of these members by small percentage $\bar{r}_{s}$ and $r_{s-1}$ respectively, i.e. from $N_{s}^{P}$ to $\left(1-\bar{r}_{s}\right) N_{s}^{P}$ and from $M_{s-1}^{P}$ to $\left(1-r_{s-1}\right) M_{s-1}^{P}$, respectively. In particular, if it is stipulated to enforce a state of sequential failure, starting from, say, joint $s$ of Fig. 2a and progressing gradually towards the supports, then it would be sufficient to assign a moment reduction quotients $\bar{r}_{s}$ to the active ends of the horizontal members meeting at $s$ and $r_{i}$ to each vertical member, such that $\bar{r}_{s}<r_{s-1}, \quad(1-$ $\left.r_{i}\right) M_{i}^{P}<\left(1-r_{i+1}\right) M_{i+1}^{P}$ and that $r_{i=0}=0$. The effects of reduction of local moments of resistance of the active members on the global carrying capacity of the girder may be assessed by comparing the magnitudes of the internal work capacities of the girder before and after RBS treatment. The compensation for such a reduction may then be included as part of the stipulated load factor. The following example has been provided to illustrate the applications of the proposed method of controlling the sequences of formation of the plastic hinges in VG of US.

\section{Demonstrative example 6}

Redesign the VG of example 1 for $n=6$ in such a way that the sequence of formation of the active plastic hinges would follow the order shown in Fig. 2a.

Solution let $r_{0}=0, r_{1}=0.05, r_{2}=0.10$ and $\bar{r}_{3}=0.15$. This reduces $M_{i}^{P}$ to $\left(1-r_{i}\right)(6-2 i) \mathrm{WL} / 4$. From Eq. (11), $W_{\text {int. }}=9 \mathrm{WL} \theta$. The effects of $r_{i}$ on the internal work may be computed as;

$$
\begin{aligned}
W_{\text {int.after }} & =4\left[\left(1-\bar{r}_{n / 2}\right) N_{n / 2}^{P}+\sum_{0}^{\frac{n}{2}-1}\left(1-r_{i}\right) M_{i}^{P}\right] \theta \\
& =9 \mathrm{WL} \theta-0.475 \mathrm{WL} \theta=8.525 \mathrm{WL} \theta
\end{aligned}
$$

This implies that the original load factor may be increased by a compensation factor of 9/8.525.

\section{Conclusions}

This article has introduced a number of simple ideas that lead to the efficient design of parallel chord steel $\mathrm{VG}$, namely, the introduction of the imaginary Basic modules, displacement-based plastic design and a method of 
computing plastic displacements at incipient collapse. An analytic procedure has been provided to facilitate and revive interest in plastic design of efficient VG. The philosophical differences between plastic design analysis and design led plastic analysis have been discussed and demonstrated through generic and numerical examples. In design led plastic analysis, collapse modes and stability conditions are imposed rather than investigated. The theory of structures is applied rather than followed.

Several numerical solutions have been presented to demonstrate the accuracy and applications of the proposed concepts and design formulae. The proposed formulae are entirely suitable for manual as well as spreadsheet and electronic computations. The proposed designs satisfy all conditions of the uniqueness theorem, and as such, cannot be far from minimum weight solutions. However, they are limited to regular vierendeel frames where all members can develop full plastic moments at collapse.

It has been demonstrated, contrary to the common belief, that displacements associated with plastic design, can be computed manually and utilized advantageously without resorting to lengthy analysis or high power computers. The proposed methodologies offer displacement control options at first yield and incipient collapse. The determination of maximum plastic displacements of the class of girders discussed in the paper has been reduced to the summation of maximum displacements of simple modules.

It has been shown that predetermined sequences of formation of plastic hinges could be arranged through rational selection of the strengths and stiffnesses of the constituent modules of the framework.

The advantage of the proposed concept over traditional methods of design is its ability to provide economic/optimized solutions while maintaining displacement control at ultimate loading. The proposed procedures are particularly useful for the preliminary design of regular steel VG.

Authors' contributions The author has been involved in structural research for well over 40 years. His latest contributions include the introduction of Performance Control as an alterative design philosophy for ductile structures.

Conflict of interest The author declares that he has no competing interests.

Open Access This article is distributed under the terms of the Creative Commons Attribution License which permits any use, distribution, and reproduction in any medium, provided the original author(s) and the source are credited.

\section{Appendix}

A brief description of the formulation of the maximum normal nodal displacements of VG of US at incipient collapse is presented in this appendix. Assuming that the girder remains kinematically stable until the formation of the last sets of plastic hinges at each side of joint $s$, then the generalized elastic-plastic normal nodal displacement of the same joint may be computed by performing the virtual work summation over all members of the framework, i.e.,

$1 \times \Delta_{s}=\sum_{i=1}^{n} M_{i} \theta_{i}+\sum_{i=2}^{n} \oint(M / E I)_{i} m_{i} d s$

where:

$$
\begin{aligned}
M_{i} & =\frac{M_{i}^{R}}{4} \text { or } M_{i}=M_{i}^{P} \text { and } m_{i} \\
& =\left[\frac{\left(\bar{L}-\bar{L}_{s}\right)}{\bar{L}}-\left\langle\frac{\bar{L}_{i}-\bar{L}_{s}}{\bar{L}}\right\rangle^{0}\right] \frac{L_{i}}{4}
\end{aligned}
$$

are the corner moments of the $i$ th module due to the external nodal forces and the unit load acting on joint $s$, respectively. Here, $\bar{L}, \bar{L}_{s}$ and $\bar{L}_{i}$ are the total span length, and distances to joint $s$ and $i$ from the left hand support, respectively, see Fig. 6b, c. It is instructive to note that $m_{i}$ can be expressed in terms of two constant multipliers and the width of each module $L_{i}$, thus:

$$
\begin{aligned}
m_{i} & =\frac{\left(\bar{L}-\bar{L}_{s}\right) L_{i}}{4 \bar{L}}=k_{\mathrm{left}} L_{i}, \text { for } 1 \leq i \leq s \text { and } m_{i}=\frac{\bar{L}_{s} L_{i}}{4 \bar{L}} \\
& =k_{\mathrm{right}} L_{i} \text { for } s+1 \leq i \leq n
\end{aligned}
$$

Since at the onset collapse, the plastic hinges associated with rotations $\theta_{i}$ are just forming, they may be set to zero to compute the desired displacement (Hayman 1961, 1971; Horne 1979). Consider the generalized curvatures caused by the racking moments of individual modules of Fig. 1d and the distribution of the virtual moments described by Eq. (A2), then the corresponding virtual work equation, assuming $\left|m_{s}\right|>\left|m_{s+1}\right|$, may be formulated as:

$$
\begin{aligned}
\Delta= & \sum_{i=1}^{s-1} \frac{4 M_{i} m_{i} L_{i}}{6 E J_{i}}\left(1+\frac{1}{\rho_{i}}\right)+\frac{2 M_{s} m_{s} L_{s}}{6 E J_{s}}\left(2+\frac{1}{\rho_{s}}\right) \\
& +\frac{h}{6 E}\left[\frac{2\left(M_{s}-M_{s+1}\right)\left(m_{s}-m_{s+1}\right)}{\left(I_{s}+I_{s+1}\right)}\right] \\
& +\frac{2 M_{s+1} m_{s+1} L_{s+1}}{6 E J_{s+1}}\left(2+\frac{1}{\rho_{s+1}}\right)+\sum_{i=s+2}^{n} \frac{4 M_{i} m_{i} L_{i}}{6 E J_{i}}\left(1+\frac{1}{\rho_{i}}\right)
\end{aligned}
$$

The complexities associated with Eq. (A4) may be alleviated by reducing it to the symbolic form;

$\Delta=\left(A_{i}+B_{s}\right) k_{\text {left }}+C_{s}\left(k_{\text {left }}-k_{\text {right }}\right)+\left(D_{s+1}+E_{i}\right) k_{\text {right }}$

where, the first and last summation terms, $A_{i}$ and $E_{i}$ describe the accumulative contributions of the individual modules to the left and right sides, respectively, of the modules that share the common vertical at joint $s$. The 

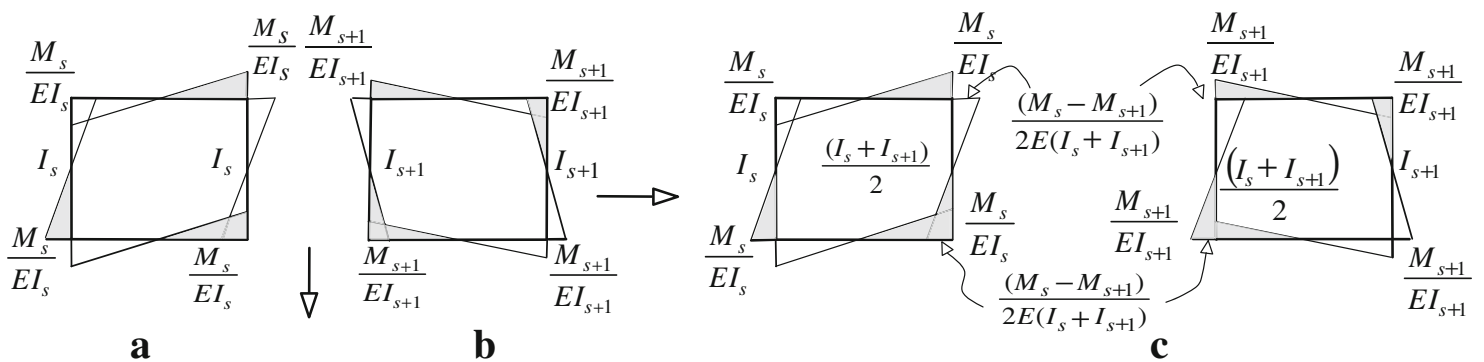

Fig. 5 a, b Curvature diagrams of modules sharing the joint of maximum displacement before merging, c curvature diagrams of the same modules after merging

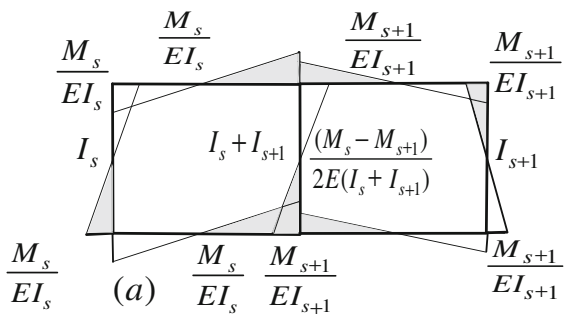

$\mathbf{a}$

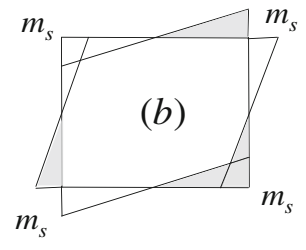

b

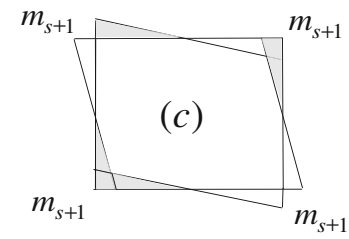

c

Fig. 6 Curvature diagrams for adjoining modules that share the same maximum displacement. a Curvature diagrams reflecting compatible deformations for the vertical members at joint $s$. b, c Unit load moments for independent modules $s$ and $s+1$, respectively

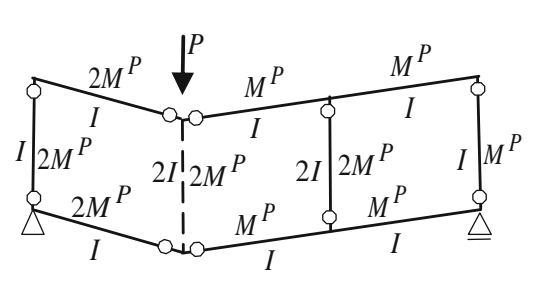

a

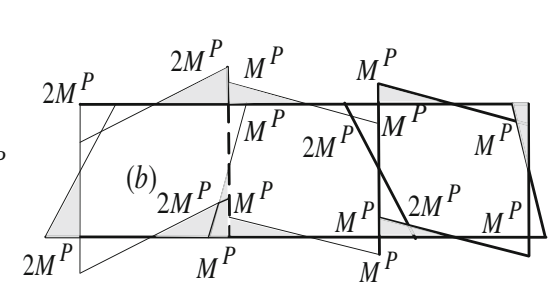

b

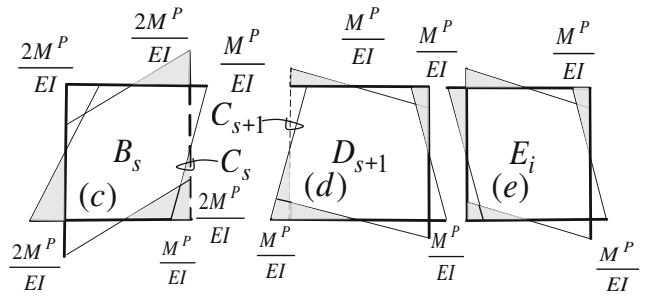

c

Fig. 7 Example 7, three bay parallel chord vierendeel girder under a single normal nodal force. a Collapse mode and properties. b Plastic bending moments. c Pictorial interpretation of Eq. (A8)

second and fourth terms, $B_{s}$ and $D_{s+1}$, describe the contributions of the, sth and $s+1$ th modules of Fig. $4 \mathrm{~d}$, respectively, less the contribution of their common vertical element at $s$. The remaining term $C_{s}$ contains the contribution of the vertical member at $s$, where the curvature changes sign. The latter term also describes the common curvature shared by modules $s$ and $s+1$. A pictorial interpretation of the compatibility conditions between the adjoining modules at $s$ is presented in Figs. 5, 6. Equation (A5) may be further simplified as:

$$
\begin{aligned}
\Delta & =\left(A_{i}+B_{s}+C_{s}\right) k_{\mathrm{left}}+\left(-C_{s}+D_{s+1}+E_{i}\right) k_{\mathrm{right}} \\
& =\Delta_{\mathrm{left}}+\Delta_{\mathrm{right}}
\end{aligned}
$$

Since $\quad k_{\text {left }}+k_{\text {right }}=1 / 4$ then $\Delta_{\text {left }}=4 k_{\text {left }} \Delta$ and $\Delta_{\text {right }}=4 k_{\text {right }} \Delta$. Therefore; $\Delta=\left(A_{i}+B_{s}+C_{s}\right) / 4$ and $\Delta=\left(-C_{s}+D_{s+1}+E_{s}\right) / 4$. Consequently;

$$
\begin{aligned}
\Delta_{\text {left }}= & \Delta=\sum_{i=1}^{s-1} \frac{M_{i} L_{i}^{2}}{6 E J_{i}}\left(1+\frac{1}{\rho_{i}}\right)+\frac{M_{s} L_{s}^{2}}{12 E J_{s}}\left(2+\frac{1}{\rho_{s}}\right) \\
& +\frac{\left(M_{s}-M_{s+1}\right) h L_{s}}{12 E\left(I_{s}+I_{s+1}\right)} \\
\Delta_{\text {right }} & =\Delta=-\frac{\left(M_{s}-M_{s+1}\right) h L_{s+1}}{12 E\left(I_{s}+I_{s+1}\right)}+\frac{M_{s+1} L_{s+1}^{2}}{12 E J_{s+1}}\left(2+\frac{1}{\rho_{s+1}}\right) \\
& +\sum_{i=s+2}^{n} \frac{M_{i} L^{2}}{6 E J_{i}}\left(1+\frac{1}{\rho_{i}}\right)
\end{aligned}
$$

Since each term of Eqs. (A7) and (A8) represents either the displacements of individual or summation of displacements of individual modules then it may be argued that; the maximum normal nodal displacements of VG of US at incipient collapse may be computed as the sum of the 
corresponding displacements of the individual modules either to the left or right of the joints of maximum plastic rotation.

Example 7: use Eqs. (A7) and (A8) to estimate the maximum normal nodal displacement of the vierendeel Girder of US of Fig. 6a at incipient collapse. $L_{1}=$ $L_{2}=L_{3}=h=L$. The moments of inertia and plastic resistance of the members are also shown in Fig. 7a.

Solution putting $\rho_{i}=1$ for $i=1,2$ and 3 and substituting for the pertinent values of $M, h, L, I$ and $J$ from Fig. 6 into Eqs. (A7) and (A8), it gives:

$$
\begin{aligned}
\Delta_{\text {left }} & =0+\frac{3 \times\left(2 M^{P}\right) L^{2}}{12 E I}+\frac{\left(2 M^{P}-M^{P}\right) L^{2}}{12 \times 2 E I} \\
& =\frac{13 M^{P} L^{2}}{24 E I} \text { and } \\
\Delta_{\text {right }} & =\frac{-\left(2 M^{P}-M^{P}\right) L^{2}}{12 \times 2 E I}+\frac{6 M^{P} L^{2}}{2 \times 12 E I}+\frac{8 M^{P} L^{2}}{2 \times 12 E I} \\
& =\frac{13 M^{P} L^{2}}{24 E I}
\end{aligned}
$$

respectively.

\section{References}

Baker ALL, Horne MR, Hayman J (1956) The Steel Skeleton, vol 1 and 2. Cambridge University Press, UK

Beedle LS (1958) Plastic design of steel frames. Wiley, USA

Bozorgnia Y, Bertero VV (2004) Earthquake engineering. CRC, Florida

Brandt AM (1984) Criteria and methods of structural optimization. Cluver Academic, USA, p 129

Foulkes J (1953) Minimum weight design and the theory of plastic collapse. Q Appl Math 10:347-358

Foulkes J (1954) The minimum-weight design of structural frames. Proc R Soc Lond Ser A 223:482-494

Goel SC, Liao WC, Bayat MR, Chao SH (2010) Performance-based plastic design (PBPD) method for earthquake resistant structures: an overview. Struct Des Tall Spec Build 19(1-2):115-137

Grigorian M (1970) Vibrations of vierendeel girders. J Sound Vib 12(4):421-427

Grigorian M (1993) On the lateral response of regular high-rise frames. Struct Des Tall build 3:2233-2252

Grigorian M (2013a) An introduction to performance control for moment frames of uniform response under lateral loading. Asian J Civ Eng 14(1):123-143

Grigorian M (2013b) A new approach to plastic design and optimization of parallel chord vierendeel girders. Int J Optim Civ Eng 3(3):371-388

Grigorian M (2014) Performance control for efficient design of double layer grids under uniform loading. Int J Adv Struct Eng. 6:52 (under press)

Grigorian M, Grigorian C (1988) Preliminary minimum weight design of moment frames under lateral loading. AISC Eng J 25:129-136

Grigorian M, Grigorian C (2011) Performance control for seismic design of moment frames. J Constr Steel Res 67:1106-1114
Grigorian M, Grigorian C (2012a) Performance control: a new elastic-plastic design procedure for earthquake resisting moment frames. J Struct Div ASCE 138(6):812-821

Grigorian M, Grigorian C (2012b) Lateral displacements of moment frames at incipient collapse. J Eng Struct 44:174-185

Grigorian M, Grigorian C (2012c) A new performance based design method for earthquake resisting moment frames. Can J Civ Eng 39:1-11

Grigorian M, Grigorian C (2012d) An introduction to the methodology of earthquake resistant structures of uniform response. Buildings 2:107-125

Grigorian M, Grigorian C (2012e) Recent developments in plastic design analysis of steel moment frames. J Constr Steel Res 76:83-92

Grigorian M, Grigorian C (2013) Drift control for multistory moment frames under lateral loading. Int J High-Rise Build 2(4):1-11

Hamburger RO, Krawinkler H, Malley JO, Adan SM (2009) Seismic design of steel special moment frames: NEHRP seismic design technical brief no. 2, National Institute of Standards and Technology, Gaithersburg, MD, NIST GCR 09-917-3

Hayman J (1961) On the estimation of deflections in elastic plastic frames. Proc Inst Civ Eng 19:89

Hayman J (1971) Plastic design of frames. Cambridge University Press, UK

Hendry AW (1955) Plastic analysis and design of mild steel vierendeel girders. Struct Eng 33:7

Horne MR (1979) Plastic theory of structures. Pergamon, London

Mazzolani MM, Piluso V (1997) Plastic design of seismic resistant steel frames. Earthq Eng Struct Dyn 26(2):167-191

Morovich AP (1958) Applicability of plastic design to the vierendeel trusses. Tulane University of Louisiana, USA

Neal BG (1963) The plastic methods of structural analysis. Chapman and Hall, London

Nethercot DA (2001) Limit state design of structural steelwork. Spon, UK

Taranath BS (1998) Steel, concrete, and composite design of tall buildings, 2nd edn. McGraw Hill, USA, pp 714-717

Wong MB (2009) Plastic analysis and design of steel structures. Elsevier, UK

Mark Grigorian The author is an international consultant, investigator and lecturer. He has more than 45 years of experience in structural design, education, code and standard development, forensic studies, research and project management. He holds B.Sc. and M.Sc. degrees in Structural Engineering from the University of Manchester (UMIST) and a D.Phil. degree in engineering from the University of Oxford. He is a California licensed Civil and Structural Engineer and is an active member of the Structural Engineers association of Southern California (SEAOC). He is a founding member and the first chairman of the Faculty of Structural Engineering (Sazeh) of Sharif University in Iran. Currently he is the Chief Structural Engineer of MGA Structural Engineering Consultants Inc. based in Glendale Ca. In addition to his technical activities, he maintains an active social role that focuses on Martial Arts and Human Behavior. His latest books, In Search of the Mind, and Karate-Do a Way of Life, deal mainly with behavioral aspects of human life and social conditions. He can be reached at (818)-242-6475 and (818)261-2133, E-mail, markarjan@aol.com. Correspondence address 1589 Trenton Ave. Glendale, Ca. 91206, US. 